\section{Question time for psychologists}

\author{
Stuart Sutherland
}

Stevens' Handbook of Experimental Psychology, 2nd edn. Vol. 1 Perception and Motivation; Vol. 2 Learning and Cognition. Edited by Richard C. Atkinson, Richard J. Herrnstein, Gardner Lindzey and R. Duncan Luce. Wiley: 1988. Vol. 1 pp. 905, \$85, £77.50. Vol. 2 pp. 1,027. \$95, $£ 85$. Two-volume set $\$ 180, £ 160$.

IN PSYCHOLOGY, old problems never die, they only fade away. On the other hand, new problems emerge at a remarkable rate. So it is instructive to compare the second edition of Stevens' Handbook of Experimental Psychology with the first, which was written nearly 30 years earlier.

The 'phi phenomenon', the illusion of movement occurring without anything being seen to move, received a great deal of attention in the first edition, but is not so much as mentioned in the second. As to the new and largely unanswered problems, here are two. First, when an ambiguous word (such as 'bank') is encountered, do people unconsciously access both meanings of it or are they directly guided by the context to the correct interpretation? Second, it has recently been discovered that people are very bad at probabilistic reasoning. For example, they fail to obey Bayes' law since they attach far too little weight to prior probabilities. Why this should be so remains unclear, although it may in part be due to a tendency to access the most salient material. For example, most people wrongly believe there are more words beginning with ' $r$ ' than words having ' $r$ ' as a second letter, because it is easier to recover from memory words starting with ' $r$ ' than words having ' $r$ ' in any other position.

\section{Stereopsis}

The failure to solve old problems and the constant emergence of new ones suggests that psychology makes little progress, but this is not entirely true. For example, in stereopsis the task of deciding which points on each eye are being stimulated by the same points in space (the correspondence problem) is an ancient one, but there are now plausible algorithms that can execute it. These receive only a cursory mention in the chapter on visual perception: unfortunately some psychologists still regard workers in artificial intelligence as rivals rather than allies.

Although the most secure advances may have occurred in vision and hearing, many discoveries have been made and a few theories put forward in other areas that are unlikely to be completely swept away. The notion that a concept may be based on a prototypical instance to which other instances bear a family resemblance goes back to Wittgenstein, but more recently it has been experimentally shown to apply to at least some concepts. As Eliot Hearst shows in an exceptionally clear and thorough chapter, even animal learning, currently a neglected field, has taken a dramatic step forward. It has been found that the formation of an association between two stimuli (for example, the conditioned and unconditioned stimuli) depends not merely on how frequently one is followed by the other, but on the second stimulus occurring more frequently after the first than in its absence. From a functional standpoint, this makes good sense. Animals, and people, need to learn about stimuli that predict an event better than other stimuli, not merely about random connections between the members of a pair of stimuli.

In psychology, the discovery of new phenomena has usually been determined by changes in the Zeitgeist, which in turn have often been influenced by the importation of new ways of thought from other disciplines. Elderly experimental psychologists, such as myself, have seen the subject successively transformed by cybernetics, information theory, Chomsky's generative grammars and artificial intelligence. All four movements suggest that it may be possible to describe and explain complex information-processing with rigour; hence the swing of experimental psychology away from behaviourism to a cognitive approach. The word 'cognitive' has been broadly interpreted because it includes not merely conscious mental processes but the unconscious ones that underlie them. Oddly enough it is the unconscious processes on which most progress has been made. Whereas there are plausible, but unfinished, theories about object recognition (a topic completely omitted from both editions), pitch perception, auditory localization and speech perception, theorizing on the higher realms of cognition remains vague. We have very little idea how memories are accessed and a fortiori how they are organized. And although it seems certain that reasoning proceeds by manipulating the semantic content of mental models rather than by the formal application of a logical calculus, we can hardly begin to specify what these mental models are like.

The influence of the information-processing sciences on psychology is new, but that of neurophysiology and related disciplines goes back many years. Today, hacking out pieces of animals' brains more or less at random has been largely supplanted by the study of neurological patients whose brains have suffered random damage through strokes, tumours or accidents. Such research is a secure and easy route to a $\mathrm{PhD}$ or an enlarged list of papers. But although it may provide information on where in the brain a given function is localized, it rarely yields any evidence that is relevant to the really important question of the nature of the mechanisms that execute it. Again, although Bartley Hoebel provides a detailed and scholarly account of recent work on neurotransmitters, the knowledge that they are heavily implicated in a variety of motivational systems, or even the more specific knowledge that the catecholamines play a role in the 'pleasure centres', does not appear to have advanced our knowledge of the mechanisms underlying reinforcement - let alone pleasure.

\section{Experiments}

The Handbook is not for beginners. It is written with the accuracy one might expect from the 40 or so distinguished contributors, but many of the chapters will be opaque to psychologists who do not themselves work in the field under review. There is little attempt to place a topic in perspective or to state clearly which problems (if any) have been solved and which remain unsolved, or even to decide what the important issues are. Instead, the reader is too often presented with a mass of experiments unadulterated by theory. Experiments are described that were intended to support theories long since shown to be fallacious - for example, those of A.M. Collins and M.R. Quillian that purported to show that the attributes of classes are held hierarchically, so that in memory yellow would be attached to canary, but flying would be attached to a higher node (bird). With several interesting exceptions, such as the chapter by S. Glucksberg and G.A. Miller on the psychology of language, most of the contributors seem to believe that if an experiment has been conducted it must be worth reporting.

Of course, experimental psychology has always lived up to its name and has tended to place more weight on experiments than on theory. The subject is, however, at present undergoing a fifth revolution, namely the development of simulated neural nets consisting of units randomly connected together, in which learning is represented by changes in the connectivity. Such nets have been found to be capable of performing surprisingly complex tasks and have been hailed as the theoretician's paradise. Unfortunately they are unlikely to introduce theoretical rigour into psychology if only because, for the most part, nobody understands how they work.

Stuart Sutherland is Director of the Centre for Research on Perception and Cognition, Univer sity of Sussex, Brighton BN1 9QG,UK. 